\title{
ON THE CONSTANT IN THE MERTENS PRODUCT FOR ARITHMETIC PROGRESSIONS. II: NUMERICAL VALUES
}

\author{
A. LANGUASCO AND A. ZACCAGNINI
}

\begin{abstract}
We give explicit numerical values with 100 decimal digits for the constant in the Mertens product over primes in the arithmetic progressions $a \bmod q$, for $q \in\{3, \ldots, 100\}$ and $(a, q)=1$.
\end{abstract}

\section{INTRODUCTION}

In our recent paper [6] we found a new expression for the constant $C(q, a)$ defined implicitly by

$$
P(x ; q, a)=\prod_{\substack{p \leq x \\ p \equiv a \text { mod } q}}\left(1-\frac{1}{p}\right)=\frac{C(q, a)}{(\log x)^{1 / \varphi(q)}}(1+o(1))
$$

as $x \rightarrow+\infty$, where, here and throughout the present paper, $q \geq 3$ and $a$ are fixed integers with $(a, q)=1, p$ denotes a prime number, and $\varphi(q)$ is the usual Euler totient function. When $q \in\{1,2\}$ the value of $C(q, a)$ can be deduced from the classical Mertens Theorem. In particular, we proved that

$$
C(q, a)^{\varphi(q)}=e^{-\gamma} \prod_{p}\left(1-\frac{1}{p}\right)^{\alpha(p ; q, a)},
$$

where $\alpha(p ; q, a)=\varphi(q)-1$ if $p \equiv a \bmod q$ and $\alpha(p ; q, a)=-1$ otherwise, and $\gamma$ is the Euler constant. The infinite product is convergent, though not absolutely, by the Prime Number Theorem for Arithmetic Progressions.

In our paper 7 we gave a simpler proof of (2) and proved that the constants $C(q, a)$ satisfy some interesting identities but, unfortunately, these are not suitable for numerical computations. Here we derive further identities, involving Dirichlet $L$ functions, that enable us to compute numerically the values of $C(q, a)$ with many digits for comparatively small $q$. Details of these identities are given in $\$ 2$, and the results of our numerical computations are collected in 93 some sample values, truncated to 40 decimal digits, are shown in Tables [13. Finch [5] has done some numerical work in the case $q \in\{3,4\}$.

The problem of computing the values of constants defined by means of products of the form

$$
\prod_{p>A}\left(1-\frac{f_{1}(p)}{f_{2}(p)}\right)
$$

Received by the editor December 11, 2007 and, in revised form, February 12, 2008.

2000 Mathematics Subject Classification. Primary 11-04, 11Y60.

(C)2008 American Mathematical Society 
where $f_{1}$ and $f_{2}$ are monic polynomials in $\mathbb{Z}[x]$ with $\operatorname{deg}\left(f_{2}\right) \geq \operatorname{deg}\left(f_{1}\right)+2$, is very common in number theory, the most famous instance being probably the twin-prime constant. The first step is writing it as an infinite product of powers of "partial zeta functions" (see equation (4) below), which converges provided that $A$ is larger than some explicit bound. A systematic treatment of this problem can be found in Moree's paper [9], whereas earlier treatments of individual cases were given by Wrench 11 and by Lindqvist and Peetre [8, for instance. In a similar fashion, one should be able to evaluate a product such as (3), where the condition $p>A$ is replaced by $p \equiv a \bmod q$ : for example, see $\S 2.3$ of the book by Finch [4 for the case of the Landau-Ramanujan constant. This is essentially what happens for $C(q, a)$, given identity (2).

\section{THEORETICAL FRAMEWORK}

In this section we concentrate on the numerical computation of the values of the constant $C(q, a)$ for comparatively small values of $q$, starting from our formula (2), and give the theoretical framework for the results in $₫ 3$. We adhere to the notation in the books by Henri Cohen [1, 2.

We will use the following convention: for any real positive constant $A$ and for any Dirichlet $L$-function, we write

$$
L_{A}(\chi, s)=\prod_{p>A}\left(1-\frac{\chi(p)}{p^{s}}\right)^{-1}
$$

and do similarly for other Euler products. We want to compute

$$
\varphi(q) \log C(q, a)=-\gamma+\log \frac{q}{\varphi(q)}-\sum_{\substack{\chi \bmod q \\ \chi \neq \chi_{0}}} \bar{\chi}(a) \sum_{m \geq 1} \frac{1}{m} \sum_{p} \frac{\chi(p)}{p^{m}} .
$$

Notice that the last sum over $p$ is $\sim \chi(2) 2^{-m}$ when $m$ is large. We compute the sum over $p$ by Möbius inversion. Let $A$ be a fixed positive constant. Then

$$
\sum_{p} \frac{\chi(p)}{p^{m}}=\sum_{p \leq A q} \frac{\chi(p)}{p^{m}}+\sum_{k \geq 1} \frac{\mu(k)}{k} \log \left(L_{A q}\left(\chi^{k}, k m\right)\right) .
$$

Therefore

$$
\begin{aligned}
\varphi(q) \log C(q, a)= & -\gamma+\log \prod_{p \leq A q}\left(1-\frac{1}{p}\right)^{\alpha(p ; q, a)} \\
& -\sum_{\substack{\chi \bmod _{\chi \neq} \\
\chi \neq \chi_{0}}} \bar{\chi}(a) \sum_{m \geq 1} \frac{1}{m} \sum_{k \geq 1} \frac{\mu(k)}{k} \log \left(L_{A q}\left(\chi^{k}, k m\right)\right) .
\end{aligned}
$$

Grouping the terms with the same value of $k m$, we see that the last part is

$$
\sum_{m \geq 1} \frac{1}{m} \sum_{k \geq 1} \frac{\mu(k)}{k} \log \left(L_{A q}\left(\chi^{k}, k m\right)\right)=\sum_{n \geq 1} \frac{1}{n} \sum_{k \mid n} \mu(k) \log \left(L_{A q}\left(\chi^{k}, n\right)\right) .
$$

Notice that the Riemann zeta function is never computed at $s=1$ in (5D), since $k m=1$ implies $k=1$, and this in its turn implies $\chi^{k}=\chi=\chi_{0}$. For $n>1$ we use

$$
\left|\log \left(L_{A q}\left(\chi^{k}, n\right)\right)\right| \leq \frac{1}{(n-1)(A q)^{n-1}} .
$$


This inequality is a consequence of the following lemma. We remark that a stronger result is valid for small $n$, but the simple bound below suffices for our applications.

Lemma 1. Let $\chi \bmod q$ be any character and $n \geq 2$ be an integer. If $B \geq 1$ is an integer, then

$$
\left|\log \left(L_{B}(\chi, n)\right)\right| \leq \frac{B^{1-n}}{n-1} .
$$

Proof. By the triangle inequality,

$$
\begin{aligned}
\left|\log \left(L_{B}(\chi, n)\right)\right|=\left|\sum_{p>B} \sum_{m \geq 1} \frac{\chi^{m}(p)}{m p^{m n}}\right| & \leq \sum_{p>B} \sum_{m \geq 1} \frac{1}{m p^{m n}} \leq \sum_{k>B} \frac{1}{k^{n}} \\
& \leq \int_{B}^{+\infty} \frac{\mathrm{d} t}{t^{n}}=\frac{B^{1-n}}{n-1},
\end{aligned}
$$

as required.

We have thus reduced the task of the computation of $\log (C(q, a))$ to computing $\log \left(L_{A q}\left(\chi^{k}, n\right)\right)$ to 100 decimal places, say. In what follows we denote by $\chi$ a generic Dirichlet character $\bmod q$ and by $n \geq 1$ an integer.

First step: We write

$$
L_{A q}(\chi, n)=L(\chi, n) \prod_{p \leq A q}\left(1-\frac{\chi(p)}{p^{n}}\right)
$$

for a convenient value of $A$.

Second step: Reduction to primitive characters. Assume now that $\chi \bmod q$ is induced by $\chi_{f} \bmod f$, where $f$ is the conductor of $\chi$. Then we have the identity

$$
L(\chi, n)=L\left(\chi_{f}, n\right) \prod_{p \mid q}\left(1-\frac{\chi_{f}(p)}{p^{n}}\right) .
$$

In particular, we recall that if $\chi=\chi_{0} \bmod q$, then

$$
L\left(\chi_{0}, n\right)=\zeta(n) \prod_{p \mid q}\left(1-\frac{1}{p^{n}}\right) .
$$

Third step: First case. Now assume that $\chi$ is a primitive character modulo $f$ and that $\chi(-1)=(-1)^{n}$. Then, by Proposition 10.2.4 of Cohen [2, we have the explicit formula

$$
L(\chi, n)=\frac{1}{2}(-1)^{n-1+(n+e) / 2} W(\chi) \sqrt{f}\left(\frac{2 \pi}{f}\right)^{n} \frac{\overline{B_{n}(\chi)}}{n !},
$$

where $W(\chi)$ denotes the root number of $\chi$ (see Definition 2.2.25 in [1]), $e=0$ if $\chi$ is even and $e=1$ if $\chi$ is odd, and $B_{n}(\chi)$ denotes the $\chi$-Bernoulli number which, in its turn, is defined by means of the $n$-th Bernoulli polynomial $B_{n}(x)$ (see [2], Definition 9.1.1) as follows:

$$
B_{n}(\chi)=f^{n-1} \sum_{a=0}^{f-1} \chi(a) B_{n}\left(\frac{a}{f}\right) .
$$

This definition is valid both for primitive and imprimitive characters. This is the last identity of Proposition 9.4.5 in Cohen [2]. 
Third step: Second case. If $\chi$ is non-principal and $\chi(-1)=(-1)^{n+1}$, there are two possibilities.

- Use the $\chi$-Euler-MacLaurin summation formula (the number of steps is proportional to $q$, but all terms are elementary); see Cohen [2, Corollary 9.4.18.

- Use the functional equation, which is valid if $\chi$ is primitive: this would take a smaller number of steps, of the order $\asymp \sqrt{q} \log q$, but it needs the computation of the incomplete $\Gamma$-function.

For $q$ small, we use the Euler-MacLaurin summation formula. When computing $L(\chi, n)$ with $n$ large, the functional equation does not take into account the fact that $L(\chi, n)=1+\chi(2) 2^{-n}+$ very much smaller terms.

When using the Euler-MacLaurin formula we take a multiple $N$ of $q$ and for $\Re(s)>1$ write

$$
\begin{aligned}
L(\chi, s)= & \sum_{r<N} \frac{\chi(r)}{r^{s}}+B_{0}(\chi) \frac{N^{1-s}}{s-1} \\
& \quad-\frac{1}{N^{s}} \sum_{j=1}^{T} \frac{(-1)^{j-1} B_{j}(\chi)}{j !} \frac{s(s+1) \cdots(s+j-2)}{N^{j-1}}+R(T),
\end{aligned}
$$

where

$$
\begin{aligned}
R(T) & =-\frac{1}{T !} s(s+1) \cdots(s+T-1) \int_{N}^{+\infty} B_{T}\left(\chi^{-},\{t\}_{\chi}\right) \frac{\mathrm{d} t}{t^{s+T}}, \\
B_{T}\left(\chi^{-},\{t\}_{\chi}\right) & =f^{T-1} \sum_{r \bmod f} \chi^{-}(r) B_{T}\left(\left\{\frac{t+r}{f}\right\}\right)
\end{aligned}
$$

and $\chi^{-}(n)=\chi(-n)$; see Definitions 9.4.2 and 9.4.10 in 2. The asymptotic series above is not convergent: we take terms until $R(T)$ reaches a small minimum, before it starts growing again.

Notice that $B_{0}(\chi)=0$ in (8) for non-principal $\chi$ by Proposition 9.4.5 of Cohen 2 and the remarks immediately following it. This is indeed crucial for the rapidity of convergence.

When $\chi(-1)=(-1)^{n}$ we use (77) to estimate $B_{n}(\chi) \asymp n !(q /(2 \pi))^{n}$. If $\chi(-1)=$ $(-1)^{n+1}$, then $B_{n}(\chi)=0$.

Computation of the root number. If $\chi$ is a primitive character modulo $q$, then the root number $W(\chi)$ is defined by means of

$$
W(\chi)=\frac{\tau(\chi)}{\sqrt{q} i^{e}}, \quad \text { where } \chi(-1)=(-1)^{e} \text { and } e \in\{0,1\},
$$

and $\tau(\chi)=\sum_{r=1}^{q} \chi(r) e(r / q)$ is the Gauss sum. It is well known that $|W(\chi)|=1$. If $\chi^{2}=\chi_{0}$, then $\chi$ is a Legendre symbol and $W(\chi)=1$.

For $q$ small, this is all right. For $q$ large, we use the functional equation, which is valid for primitive $\chi$, introduce

$$
c(\chi)=\sum_{n \geq 1} \chi(n) e^{-\pi n^{2}}
$$

and notice that

$$
W(\chi)=\frac{c(\chi)}{i^{e} \overline{c(\chi)}} .
$$




\section{DESCRIPTION OF THE COMPUTER PROGRAM}

First of all, we need to generate the complete set of Dirichlet characters $\bmod q$ and also to compute their orders and conductors and whether they are primitive or not. To this end we follow the argument in $\S 4$ of Davenport [3]: we first generate the characters for any $p^{\alpha} \mid q$, paying particular attention to the case when $q$ is an even integer, and then we build by multiplication the characters to the modulus $p_{1}^{\alpha} p_{2}^{\beta}$ with $p_{1} \neq p_{2}$ and $p_{1}, p_{2} \mid q$. To compute the order and the primitivity of this character we use Proposition 2.1.34 of [1. The conductor of a character is obtained using the necessary and sufficient condition described in Lemma 2.1.32 of [1].

In order to evaluate (5) using a computer program we have to truncate the sums over $k$ and $m$ and to estimate the error we are introducing. Let $M, K>1$ be two integers. We have

$$
\begin{aligned}
& \log \prod_{p>A q}\left(1-\frac{1}{p}\right)^{\alpha(p ; q, a)}=-\sum_{\substack{\chi \bmod _{\chi} \\
\chi \neq \chi_{0}}} \bar{\chi}(a) \sum_{1 \leq m \leq M} \frac{1}{m} \sum_{p>A q} \frac{\chi(p)}{p^{m}} \\
& -\sum_{\substack{\chi \neq \bmod q \\
\chi \neq \chi_{0}}} \bar{\chi}(a) \sum_{m>M} \frac{1}{m} \sum_{p>A q} \frac{\chi(p)}{p^{m}} \\
& =-\sum_{\substack{\chi \neq \chi_{0} \\
\chi \neq \chi_{0}}} \bar{\chi}(a) \sum_{1 \leq m \leq M} \frac{1}{m} \sum_{1 \leq k \leq K} \frac{\mu(k)}{k} \log \left(L_{A q}\left(\chi^{k}, k m\right)\right) \\
& -\sum_{\substack{\chi \bmod q \\
\chi \neq \chi_{0}}} \bar{\chi}(a) \sum_{1 \leq m \leq M} \frac{1}{m} \sum_{k>K} \frac{\mu(k)}{k} \log \left(L_{A q}\left(\chi^{k}, k m\right)\right) \\
& -\sum_{\substack{\chi \neq \bmod q \\
\chi \neq \chi_{0}}} \bar{\chi}(a) \sum_{m>M} \frac{1}{m} \sum_{p>A q} \frac{\chi(p)}{p^{m}} \\
& =-S(q, a)-E_{1}(q, a, A, K)-E_{2}(q, a, A, M),
\end{aligned}
$$

say. Using (6) and the trivial bound for $\chi$, it is easy to see that

$$
\left|E_{1}(q, a, A, K)\right| \leq \frac{2 A q(\varphi(q)-1)}{2 K(A q-1)\left[(A q)^{K}-1\right]}
$$

and

$$
\left|E_{2}(q, a, A, M)\right| \leq \frac{A q(\varphi(q)-1)}{M(M-1)(A q-1)(A q)^{M}} .
$$

In order to ensure that $S(q, a)$ is a good approximation of $C(q, a)$, it is sufficient that $A q, K$ and $M$ are sufficiently large. Setting $A q=9600$ and $K=M=26$ yields the desired 100 correct decimal digits.

Now we have to consider the error we are introducing during the evaluation of the Dirichlet $L$-functions that appear in $S(q, a)$. Notice that in the case involving the Bernoulli numbers we use an exact formula: hence we just need to evaluate the error introduced by the $R(T)$ term in the Euler-McLaurin summation formula (8)). In fact the Euler-McLaurin summation formula is used in about $1 / 4$ of the total cases, but we are now just looking for an upper bound and so we will sum $|R(T)|$ over $m \leq M$ and $k \leq K$. 
Assume now that $T \geq 2$ is an even integer and $q \mid N$. For any non-principal character $\chi^{k} \bmod q$, equation (8) implies that

$$
\begin{aligned}
L_{T, N}\left(\chi^{k}, k m\right)= & \sum_{r<N} \frac{\chi^{k}(r)}{r^{k m}} \\
& \quad-\frac{1}{N^{k m}} \sum_{j=1}^{T} \frac{(-1)^{j-1} B_{j}\left(\chi^{k}\right)}{j !} \frac{k m(k m+1) \cdots(k m+j-2)}{N^{j-1}}
\end{aligned}
$$

and hence we get

$$
L_{A q}\left(\chi^{k}, k m\right)=\Pi\left(L_{T, N}\left(\chi^{k}, k m\right)-E_{3}\left(q, m, k, N, T, \chi^{k}\right)\right),
$$

where $\Pi$ denotes the finite products we wrote in the first and second step of \$2. Moreover it is clear that

$$
\begin{aligned}
& \left|E_{3}\left(q, m, k, N, T, \chi^{k}\right)\right| \\
& \quad \leq \frac{k m(k m+1) \cdots(k m+T-1)}{T !} \int_{N}^{+\infty}\left|B_{T}\left(\left(\chi^{k}\right)^{-},\{t\}_{\chi^{k}}\right)\right| t^{-k m-T} \mathrm{~d} t .
\end{aligned}
$$

Hence

$$
\left|\log \left(L_{A q}\left(\chi^{k}, k m\right)\right)-\log \left(\Pi \cdot L_{T, N}\left(\chi^{k}, k m\right)\right)\right| \leq\left|\frac{E_{3}\left(q, m, k, N, T, \chi^{k}\right)}{L_{T, N}\left(\chi^{k}, k m\right)}\right|
$$

and the total error arising in the computation of the Dirichlet $L$-functions can be obtained by summing the previous estimate over $m$ and $k$. For $T$ even, trivial estimates and Proposition 9.1.3 of [2] imply that

$$
\begin{aligned}
\left|B_{T}\left(\left(\chi^{k}\right)^{-},\{t\}_{\chi^{k}}\right)\right| & \leq f^{T-1} \sum_{r \bmod f}\left|\chi^{k}(-r) B_{T}\left(\left\{\frac{t+r}{f}\right\}\right)\right| \\
& \leq f^{T-1} \sum_{r \bmod f}\left|\sum_{j=0}^{T}\left(\begin{array}{c}
T \\
j
\end{array}\right) B_{j}\left\{\frac{t+r}{f}\right\}^{T-j}\right| \leq f^{T} B_{T},
\end{aligned}
$$

where $f \mid q$ is the conductor of $\chi^{k}$ and $B_{T}$ is the $T$-th Bernoulli number. Hence we obtain

$$
\begin{aligned}
\left|E_{3}\left(q, m, k, N, T, \chi^{k}\right)\right| & \leq \frac{k m(k m+1) \cdots(k m+T-1) q^{T} B_{T}}{T !} \frac{N^{1-k m-T}}{k m+T-1} \\
& =\frac{q^{T} B_{T}}{T !}(k m) \cdots(k m+T-2) N^{1-k m-T} .
\end{aligned}
$$

Moreover, let

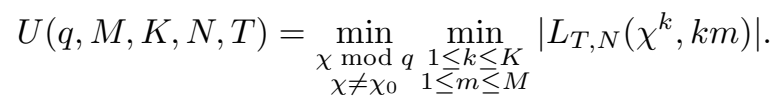


The total error arising in the computation of the Dirichlet $L$-functions is therefore

$$
\begin{aligned}
\mid E_{4} & (q, a, M, K, N, T) \mid \\
& \leq \frac{(\varphi(q)-1) q^{T} B_{T}}{U(q, M, K, N, T)} \sum_{1 \leq m \leq M} \frac{1}{m} \sum_{1 \leq k \leq K} \frac{1}{k} \frac{(k m) \cdots(k m+T-2)}{T !} N^{1-k m-T} \\
& =\frac{(\varphi(q)-1) q^{T} B_{T}}{U(q, M, K, N, T) T !} \sum_{1 \leq m \leq M} \sum_{1 \leq k \leq K}(k m+1) \cdots(k m+T-2) N^{1-k m-T} \\
& \leq \frac{(\varphi(q)-1)(K M+T-2)^{T-2} q^{T} B_{T}}{U(q, M, K, N, T) N^{T-1} T !} \sum_{1 \leq m \leq M} \sum_{1 \leq k \leq K} N^{-k m} \\
& \leq \frac{2(\varphi(q)-1)(K M+T-2)^{T-2} q^{T} B_{T}}{(N-1) U(q, M, K, N, T) N^{T-1} T !} .
\end{aligned}
$$

Letting

$$
\widetilde{C}(q, a)=\left(e^{-\gamma} \prod_{p \leq A q}\left(1-\frac{1}{p}\right)^{\alpha(p ; q, a)} \exp (-S(q, a))\right)^{1 / \varphi(q)}
$$

and collecting the previous estimates, we have that

$$
\begin{aligned}
|C(q, a)-\widetilde{C}(q, a)| & \leq \widetilde{C}(q, a)\left|\exp \left(-\frac{E(q, a, A, M, K, N, T)}{\varphi(q)}\right)-1\right| \\
& \leq \widetilde{C}(q, a) \frac{|E(q, a, A, M, K, N, T)|}{\varphi(q)}
\end{aligned}
$$

where $E(q, a, A, M, K, N, T)$ denotes $E_{1}(q, a, A, K)+E_{2}(q, a, A, M)+E_{4}(q, a$, $M, K, N, T)$.

Summing up, the final error we have in computing $C(q, a)$ as $\widetilde{C}(q, a)$ is

$$
E_{\text {final }}(q, a, A, K, M, T, N) \leq \widetilde{C}(q, a) \frac{|E(q, a, A, M, K, N, T)|}{\varphi(q)} .
$$

Practical experimentations for $q \in\{3, \ldots, 100\}$ suggested to use different ranges for $N$ and $T$ to reach a precision of at least 100 decimal digits in a reasonable amount of time. Using $A q=9600, M=K=26$ and recalling that $q \mid N$ and $T$ is even, our choice is $N=(\lfloor 16800 / q\rfloor+1) q$ and $T=88$ if $q \in\{3, \ldots, 10\}$, while for $q \in\{90$, $\ldots, 100\}$ we have to use $N=(\lfloor 40320 / q\rfloor+1) q$ and $T=204$. Intermediate ranges are used for the remaining integers $q$.

The programs we used to compute the Dirichlet characters $\bmod q$ and the values of $C(q, a)$ for $q \in\{3, \ldots, 100\}, 1 \leq a \leq q,(a, q)=1$, were written using the GP scripting language of PARI/GP [10; the C program was obtained from the GP one using the gp2c tool. The actual computations were performed using several LinuX PCs and one Apple MacMini computer for a total amount of computing time equal to 1897.036096 hours $=79.043171$ days.

A tiny part of the final results is collected in the following tables. The complete set of results can be downloaded from www.math.unipd.it/ ${ }^{\text {languasc/MCcomput. }}$ html together with the source program in GP and the results of the verifications of the identities (9) and (10) which are described in the section below. 


\section{Verification OF CONSISTENCY}

The set of constants $C(q, a)$ satisfies many identities, and we checked our results verifying that these identities hold within a very small error. The basic identities that we exploited are two: the first one is

$$
\prod_{\substack{a \bmod q \\(a, q)=1}} C(q, a)=e^{-\gamma} \frac{q}{\varphi(q)} .
$$

This can be verified using either the definition (1) or the identity (2), taking into account the fact that primes dividing $q$ do not occur in any of the products $P(x ; q, a)$.

The other identity is valid whenever we take two moduli $q_{1}$ and $q_{2}$ with $q_{1} \mid q_{2}$ and $\left(a, q_{1}\right)=1$. In this case we have

$$
C\left(q_{1}, a\right)=\prod_{\substack{j=0 \\\left(a+j q_{1}, q_{2}\right)=1}}^{n-1} C\left(q_{2}, a+j q_{1}\right) \prod_{\substack{p \mid q_{2} \\ p \equiv a \bmod q_{1}}}\left(1-\frac{1}{p}\right),
$$

where $n=q_{2} / q_{1}$. The proof depends on the fact that the residue class $a \bmod q_{1}$ is the union of the classes $a+j q_{1} \bmod q_{2}$, for $j \in\{0, \ldots, n-1\}$. If $q_{1}$ and $q_{2}$ have the same set of prime factors, the condition $\left(a+j q_{1}, q_{2}\right)=1$ is automatically satisfied, since $\left(a, q_{1}\right)=1$ by our hypothesis. On the other hand, if $q_{2}$ has a prime factor $p$ that $q_{1}$ lacks, then there are values of $j$ such that $p \mid\left(a+j q_{1}, q_{2}\right)$ and the corresponding value of $C\left(q_{2}, a+j q_{1}\right)$ in the right-hand side of (10) would be undefined. The product at the far right takes into account these primes.

To prove (10), let $P(x ; q, a)$ be defined by the relation on the far left of (10), without restrictions on $q$ and $a$. Then, for $\left(a, q_{1}\right)=1$ and $x \geq q_{2}$, write

$$
P\left(x ; q_{1}, a\right)=\prod_{j=0}^{n-1} P\left(x ; q_{2}, a+j q_{1}\right)=\prod_{\substack{j=0 \\\left(a+j q_{1}, q_{2}\right)=1}}^{n-1} P\left(x ; q_{2}, a+j q_{1}\right) \Pi\left(x ; q_{2}, q_{1}, a\right),
$$

say. The primes $p \leq x$ such that $p \equiv a \bmod q_{1}$ and $p \nmid q_{2}$ appear in the product in the right-hand side above, since there is exactly one value of $j$ such that $p \equiv$ $a+j q_{1} \bmod q_{2}$ and for any such prime it is obvious that $\left(a+j q_{1}, q_{2}\right)=1$. The only primes that are left are those lying in the residue class $a \bmod q_{1}$ and that divide $q_{2}$. Hence $\Pi\left(x ; q_{2}, q_{1}, a\right)$ is exactly the product on the far right of (10). Now (10) follows from multiplying by a suitable power of $\log x$ and taking the limit as $x \rightarrow+\infty$.

The validity of (9) was checked immediately at the end of the computation of the constants $C(q, a)$, for a fixed $q$ and for every $1 \leq a \leq q$ with $(a, q)=1$ by the same program that computed them. These results were collected in a file, and a different program checked that (10) holds within a very small error by building every possible relation of that kind for every $q_{2} \in\{3, \ldots, 100\}$ and $q_{1} \mid q_{2}$ with $1<q_{1}<q_{2}$. The total number of identities checked is

$$
\sum_{q=3}^{100} \sum_{\substack{d \mid q \\ 1<d<q}} \varphi(d)=\sum_{q=3}^{100}(q-1-\varphi(q))=1907 .
$$

These identities are not independent of one another, but we did not bother to eliminate redundancies since the total time requested for this part of the computation 
is absolutely negligible. The number of independent identities is

$$
\sum_{q=3}^{100} \sum_{\substack{p \mid q \\ p<q}} \varphi\left(\frac{q}{p}\right)=\sum_{n=2}^{100} \pi\left(\frac{100}{n}\right) \varphi(n)=1408,
$$

where $p$ denotes a prime in the sum on the left.

TABLE 1. Some numerical results: the first column contains the modulus $q$, the second the residue class $a$, the third the computed value of $C(q, a)$, and the fourth is the number of correct decimal digits we obtained. The table shows the values truncated to 40 decimal digits.

\begin{tabular}{|c|c|c|c|}
\hline$q$ & $a$ & $C(q, a)$ & digits \\
\hline 3 & 1 & $1.4034774468278563951360958591826816440307 \ldots$ & 104 \\
3 & 2 & $0.6000732161773216733074128367849176047200 \ldots$ & 104 \\
4 & 1 & $1.2923041571286886071091383898704320653429 \ldots$ & 104 \\
4 & 3 & $0.8689277682343238299091527791046529122939 \ldots$ & 104 \\
5 & 1 & $1.2252384385390845800576097747492205275405 \ldots$ & 103 \\
5 & 2 & $0.5469758454112634802383012874308140377519 \ldots$ & 104 \\
5 & 3 & $0.8059510404482678640573768602784309320812 \ldots$ & 104 \\
5 & 4 & $1.2993645479149779881608400149642659095025 \ldots$ & 103 \\
$\vdots$ & $\vdots$ & $\vdots$ & $\vdots$ \\
9 & 1 & $1.1738495868654491902701394683919739604995 \ldots$ & 103 \\
9 & 2 & $0.5455303829342851960446307443914437164832 \ldots$ & 104 \\
9 & 4 & $1.1336038613343693249917335959075962374233 \ldots$ & 103 \\
9 & 5 & $0.9412310917798332515572574704874703583166 \ldots$ & 103 \\
9 & 7 & $1.0547066156548587451082819988401491024340 \ldots$ & 103 \\
9 & 8 & $1.1686623008402869661248081381642176283145 \ldots$ & 103 \\
$\vdots$ & $\vdots$ & $\vdots$ & $\vdots$ \\
15 & 1 & $1.1617073088517756555676638861655356817964 \ldots$ & 103 \\
15 & 2 & $0.5531662836641193792434413294289420522197 \ldots$ & 104 \\
15 & 4 & $1.1368510737193937042392719219836177668605 \ldots$ & 103 \\
15 & 7 & $0.9888090824844727678176951687669703243697 \ldots$ & 103 \\
15 & 8 & $1.1248826700801117041084787027689447040760 \ldots$ & 103 \\
15 & 11 & $1.0546877248711663022320456767412694068618 \ldots$ & 103 \\
15 & 13 & $1.0747134726382660587745323674368168616132 \ldots$ & 103 \\
15 & 14 & $1.1429505393911402552425384830238885435764 \ldots$ & 103 \\
$\vdots$ & $\vdots$ & $\vdots$ & $\vdots$ \\
21 & 1 & $1.1141670280743936828731735756576813156065 \ldots$ & 103 \\
21 & 2 & $0.5383301255587159174351133305605833477678 \ldots$ & 104 \\
21 & 4 & $1.1185837991946284893102162561180399170905 \ldots$ & 103 \\
21 & 5 & $0.8804463747350350872193530732768812838973 \ldots$ & 103 \\
21 & 8 & $1.0809444954913878156248769107211013330026 \ldots$ & 103 \\
21 & 10 & $1.0855302392682037293388720447231438521276 \ldots$ & 103 \\
21 & 11 & $1.0128344672130266463968855892485398266065 \ldots$ & 103 \\
21 & 13 & $1.0371155725767642823358797876916780548258 \ldots$ & 103 \\
21 & 16 & $1.1035547306497255785825571380877055652196 \ldots$ & 103 \\
21 & 17 & $1.0486412692857397440465915448981610476825 \ldots$ & 103 \\
21 & 19 & $1.0574758123265342759648524359814135750529 \ldots$ & 103 \\
21 & 20 & $1.1027671924237418176511972126578877947364 \ldots$ & 103 \\
\hline
\end{tabular}


TABLE 2. Some numerical results: the first column contains the modulus $q$, the second the residue class $a$, the third the computed value of $C(q, a)$, and the fourth is the number of correct decimal digits we obtained. The table shows the values truncated to 40 decimal digits.

\begin{tabular}{|c|c|c|c|}
\hline$q$ & $a$ & $C(q, a)$ & digits \\
\hline 39 & 1 & $1.0558043473142841979273107487867952159449 \ldots$ & 103 \\
39 & 2 & $0.5203026628809482277529964233919621231701 \ldots$ & 103 \\
39 & 4 & $1.0467551202397323195593324251885584436643 \ldots$ & 103 \\
39 & 5 & $0.8477108709928609050405112584700448177533 \ldots$ & 103 \\
39 & 7 & $0.9131634445753290856338897033232908456824 \ldots$ & 103 \\
39 & 8 & $1.0491976120090375508070956898591030898489 \ldots$ & 103 \\
39 & 10 & $1.0644889181790139210569905090072544013982 \ldots$ & 103 \\
39 & 11 & $0.9611802851802015744645440449091664544815 \ldots$ & 103 \\
39 & 14 & $1.0471282217602293552090665345631733882042 \ldots$ & 103 \\
39 & 16 & $1.0694449785599316393966557136726680120488 \ldots$ & 103 \\
39 & 17 & $1.0027080336857767080150127190485342860222 \ldots$ & 103 \\
39 & 19 & $1.0063790089466405557887479935647072297591 \ldots$ & 103 \\
39 & 20 & $1.0467993224064620442361201103591601719183 \ldots$ & 103 \\
39 & 22 & $1.0521884311669460927257333479303503936214 \ldots$ & 103 \\
39 & 23 & $1.0114747946261577434516887836293420101981 \ldots$ & 103 \\
39 & 25 & $1.0597693417994788378992764465883123963780 \ldots$ & 103 \\
39 & 28 & $1.0529671095629036217092386664444649064610 \ldots$ & 103 \\
39 & 29 & $1.0267423753797454160121131413618162768076 \ldots$ & 103 \\
39 & 31 & $1.0297283934645776984576326942483733223668 \ldots$ & 103 \\
39 & 32 & $1.0482866374125031516972329668035300513497 \ldots$ & 103 \\
39 & 34 & $1.0472581549429544593781995140831083054063 \ldots$ & 103 \\
39 & 35 & $1.0562593819557667826211305540931669587921 \ldots$ & 103 \\
39 & 37 & $1.0385638656749415055234884100430210797446 \ldots$ & 103 \\
39 & 38 & $1.0674150481593719996424991312912670083485 \ldots$ & 103 \\
\hline
\end{tabular}


TABLE 3. Some numerical results: the first column contains the modulus $q$, the second the residue class $a$, the third the computed value of $C(q, a)$, and the fourth is the number of correct decimal digits we obtained. The table shows the values truncated to 40 decimal digits.

\begin{tabular}{|c|c|c|c|}
\hline$q$ & $a$ & $C(q, a)$ & digits \\
\hline 84 & 1 & $1.0762168747360169189445984481112147917766 \ldots$ & 103 \\
84 & 5 & $0.8423464320992898808305526411222358430753 \ldots$ & 103 \\
84 & 11 & $0.9670462929845278524311619985091112662169 \ldots$ & 103 \\
84 & 13 & $0.9746953940834972813365085898448043371424 \ldots$ & 103 \\
84 & 17 & $0.9978335235521385853486954919220491056500 \ldots$ & 103 \\
84 & 19 & $1.0042721918535182457015722654932145385404 \ldots$ & 103 \\
84 & 23 & $1.0128902359146896167524723309894756202191 \ldots$ & 103 \\
84 & 25 & $1.0625109746049189658962532302336200526631 \ldots$ & 103 \\
84 & 29 & $1.0217856732501917185719533836132834670012 \ldots$ & 103 \\
84 & 31 & $1.0324778423499473481419749332801549343076 \ldots$ & 103 \\
84 & 37 & $1.0448633446823406686188909998297275362347 \ldots$ & 103 \\
84 & 41 & $1.0483511545557197512968002104563579599259 \ldots$ & 103 \\
84 & 43 & $1.0352625518417795493214543003655548678836 \ldots$ & 103 \\
84 & 47 & $1.0452307283367875092541042542165185077145 \ldots$ & 103 \\
84 & 53 & $1.0473484822398583732227792995221792774100 \ldots$ & 103 \\
84 & 55 & $1.0640407032516661060398721577715786126086 \ldots$ & 103 \\
84 & 59 & $1.0509180585081298515408918851194537493615 \ldots$ & 103 \\
84 & 61 & $1.0529772913206146375443030010561915545034 \ldots$ & 103 \\
84 & 65 & $1.0629584657266981779431184953028111293016 \ldots$ & 103 \\
84 & 67 & $1.0527738780397628191309530077617335181157 \ldots$ & 103 \\
84 & 71 & $1.0578974865179095395282678213164071324168 \ldots$ & 103 \\
84 & 73 & $1.0513835694502728488866738616694632680665 \ldots$ & 103 \\
84 & 79 & $1.0561713512739106221130859861434109044623 \ldots$ & 103 \\
84 & 83 & $1.0519063079499187595778933301342325552061 \ldots$ & 103 \\
\hline
\end{tabular}




\section{ACKNowledgments}

We would like to express our warmest thanks to Henri Cohen for an illuminating discussion.

We also wish to thank K. Belabas and B. Allombert for their hints about PARI/GP and gp2c and L. Righi for his help using the PCs of the NumLab laboratory www.numlab.math.unipd.it of the Department of Pure and Applied Mathematics of the University of Padova. We also wish to thank C. Fassino for having read a preliminary version of this paper.

\section{REFERENCES}

[1] H. Cohen. Number Theory. Volume 1: Tools and Diophantine Equations, volume 239 of Graduate Texts in Mathematics. Springer, 2007.

[2] H. Cohen. Number Theory. Volume 2: Analytic and Modern Tools, volume 240 of Graduate Texts in Mathematics. Springer, 2007.

[3] H. Davenport. Multiplicative Number Theory. Springer-Verlag, third edition, 2000. MR1790423 (2001f:11001)

[4] S. R. Finch. Mathematical constants, volume 94 of Encyclopedia of Mathematics and its Applications. Cambridge University Press, Cambridge, 2003. MR 2003519 (2004i:00001)

[5] S. R. Finch. Mertens' formula. Preprint. http://algo.inria.fr/csolve/mrtns.pdf, 2007.

[6] A. Languasco and A. Zaccagnini. A note on Mertens' formula for arithmetic progressions. $J$. Number Theory, 127:37-46, 2007. MR2351662

[7] A. Languasco and A. Zaccagnini. On the constant in the Mertens product for arithmetic progressions. I. Identities. ArXiv website, 2007. arXiv:0706.2807.

[8] P. Lindqvist and J. Peetre. On the remainder in a series of Mertens. Expositiones Math., 15:467-478, 1997. MR 1486409 (98i:11110)

[9] P. Moree. Approximation of singular series and automata. Manuscripta Math., 101:385-399, 2000. MR.1751040 (2001f:11204)

[10] The PARI Group, Bordeaux. PARI/GP, version 2.3.1-2.3.2, 2005. Available from http:// pari.math.u-bordeaux.fr/

[11] J. W. Wrench, Jr. Evaluation of Artin's constant and the twin-prime constant. Math. Comp., 15:396-398, 1961. MR0124305 (23:A1619)

Dipartimento di Matematica Pura e Applicata, Università di Padova, Via Trieste 63, 35121 PADOVA, ITALY

E-mail address: languasco@math.unipd.it

Dipartimento di Matematica, Università di Parma, Parco Area delle Scienze, 53/a, Campus Universitario, 43100 Parma, Italy

E-mail address: alessandro.zaccagnini@unipr.it 